\title{
The knee joint in sports medicine
}

\author{
Albert van Kampen
}

Received: 2 January 2013 / Accepted: 2 January 2013 / Published online: 11 January 2013

(C) Springer-Verlag Berlin Heidelberg 2013

With the increase in sports participation an equal increase in sports-related injuries has been observed. Over the last few decades injuries of the knee joint have played a major role, due to many popular knee pivoting sports including soccer, skiing and basketball. Treatment options for anterior cruciate ligament (ACL) injuries have dramatically changed over the last 50 years, as described by Chambat et al. [1], which is the first invited paper in this special issue. Especially for younger orthopaedic surgeons who mainly learn to treat ACL insufficiency by arthroscopic means the development of our thoughts about the surgical treatment of this particular ruptured ligament, e.g. by the change from outside to inside the joint, from augmentation to reconstruction of the ligament itself, has been an interesting journey. Moreover, sometimes older techniques may be of considerable value in certain severe instabilities, to aid attainment of optimal stability. I refer to the articles by Vadalà et al. [2] and Dejour et al. [3] who both use an extraarticular augmentation to increase the stability. Maybe this will become a new trend in the coming years.

Another issue is the graft to use for ACL reconstruction. There has been a shift from bone-patellar tendon-bone towards the hamstrings in recent years. In some countries, for example The Netherlands, the hamstring tendons are practically exclusively used nowadays.

This shift is not based on sound scientific evidence that hamstrings perform better, actually several meta-analyses show they perform more or less equally well, but may be partly industry driven. This trend is also seen in the international survey performed by Chechik at al. [4] in this issue. Interestingly this shift is not clearly seen among the

\footnotetext{
A. van Kampen $(\square)$

Department of Orthopaedics, Radboud University Nijmegen

Medical Centre, Nijmegen, The Netherlands

e-mail: A.vanKampen@orthop.umcn.nl
}

members of the ACL Study Group. The ACL Study Group was organised around 28 years ago by a small group of orthopaedic surgeons with a common interest in the ligament. Today the group has about 180 dedicated knee surgeons as members from all over the world who meet every two years to exchange information. Every two years the members fill out a questionnaire concerning their methods of treatment. The 2012 results show that the hamstrings are used in $56 \%$ and bone-patellar tendon-bone in $35 \%$ of the reconstructions. Since the use of hamstrings has become quite popular as a graft and we all know that the quality of a single semitendinous tendon sometimes is questionable, the question arises whether it is harmful to also harvest the gracilis tendon from the same side. In an elegant study Barenius at al. [5] give us the answers. And what about patellar tendinitis after ACL surgery with the patellar tendon? Or without any surgery? Although I have the impression that this was an issue during the past few years when we used bone blocks of at least $25 \mathrm{~mm}$ in length; since that has changed to bone blocks with a maximum of $15 \mathrm{~mm}$ (personal experience) this complication hardly ever occurs. Since it seems to be more related to open chain exercises it is seen in different patients with or without surgery and with hamstrings or patellar tendon.

The use of a double bundle for ACL reconstructions is another example of a change in surgical technique to increase the final result in stability of the knee joint. Although the idea of a double bundle sounds attractive, based on the multi-ligamentous tissue of the original ACL, the main benefit of this new technique has been the awareness of the fact that an anatomical reconstruction of the ACL within the femoral footprint is not the same as an isometric reconstruction as performed for years by many surgeons. Increased stability by this new technique, however, can only be proven by comparison of double-bundle vs anatomical single-bundle reconstruction. The isometric reconstructions 
should not be included in such a comparison of course. By carefully reading the latest meta-analysis by $\mathrm{Li}$ et al. [6] and the review of Suomalainen et al. [7], it is clear that no important differences were found. Also, using new techniques such as computer navigation in a comparative study did not show significant differences with regard to one technique [8]. Therefore, for now the question whether double-bundle reconstruction will be the gold standard for the coming years remains. Interesting in this view is the very conservative view of the members of the ACL Study Group: Only $15 \%$ of the members routinely performed doublebundle reconstructions in 2012 and $85 \%$ felt that doublebundle reconstructions did not perform better! Of course there will be indications when the technique of a doublebundle reconstruction instead of a single-bundle reconstruction should be considered. Therefore, in this issue we focus on indications and contraindications for both techniques in a paper from the Pittsburgh group [9]. It is interesting to read how - in time-indications are set and changed today in comparison to a couple of years ago.

Since it is clear that there remains a broad indication to use single-bundle grafts for ACL reconstruction a renewed focus on anatomical placement and the surgical technique to reach this anatomical footprint is valuable. We have known for several years that it is impossible to properly reach the femoral footprint through the tibial tunnel; therefore, techniques have been developed using an extra medial portal, retrograde drilling of the tunnel and double-incision techniques. I invited Marcus Arnold and co-workers [10] to describe their surgical double-incision technique with bonepatellar tendon-bone as a graft and Charlie Brown and colleagues [11] to cover the issue of the medial portal technique. Both have described and beautifully illustrated the pearls of these surgical procedures.

And what about long-term results? We know that with a good position of the ACL graft, this graft will survive for a long time. However, up till now we are not able to prevent arthritis of the knee joint, probably due to the fact that we are not yet able to mimic the original ACL closely enough. The two papers in this issue with ten years follow-up, both from Germany $[12,13]$, show a significant number with established arthritis. This reminds us of the indication for surgery which should in general include the restoration of knee stability, the prevention of meniscal damage but no prevention of arthritis. And what about the possibilities for return to sport after the reconstruction? After reading the article by Julian Feller from Australia [14], I have the feeling we should also be more reluctant about sports participation at a high level. We also know from all long-term follow-up studies the importance of retaining the menisci. Sometimes retention of the meniscus is not possible, or a meniscus has been taken out already. What about synthetic replacement? How far are we today? In a review paper by
Vrancken et al. [15] we are informed of the current possibilities, results and drawbacks.

We are all more interested in success than failure, including complications. Sometimes the failure is established easily by physical examination and X-rays which show a definitively incorrect position of bone tunnels. Sometimes it is more subtle. Michael Hirschmann and co-workers [16] studied the value of single photon emission computed tomography/computed tomography (SPECT/CT), a rather new modality in medicine, in relation to graft orientation. In my view this modality might open up new ways of detecting fine abnormalities which may elucidate persistent complaints in patients.

The use of allografts and synthetics is limited in many countries. However, they are still in use and it was decided also in this issue to pay attention to these ways of reconstruction of the ACL. Although it is often stated that the rerupture rate is higher in allograft reconstructions, (I hear numbers in the range of $10 \%$ at congresses) $\mathrm{Hu}$ et al. [17] could not establish this in their meta-analysis of prospective studies. Are the more negative aspects of these allografts maybe not published? A systematic review about synthetics is included concerning the ligament augmentation and reconstruction system (LARS) [18]. An important warning looking at the results is the fact that only nine papers could be found and that all papers showed a poor methodological quality. Also included is the study from Struewer et al. [19] who showed the findings of second-look arthroscopies (more than $30 \%$ synovial reactions) after reconstruction with the LARS. After studying both papers I suppose the individual orthopaedic surgeon will draw his/her own conclusions and know which graft sources to use or not to use.

At the end of this special issue we included the paper of Boutefnouchet et al. [20]. This paper was selected because of the excellent results this group has with an arthroscopic technique of posterior cruciate ligament reconstruction.

I hope and expect that all articles in this issue will be valuable and that this issue will serve as a reference for the coming years in the treatment variability of knee injuries.

\section{References}

1. Chambat P, Guier Ch, Sonnery-Cottet B et al (2013) The evolution of ACL reconstruction over the last 50 years. Int Orthop 37(2). doi:10.1007/s00264-012-1759-3

2. Vadalà AP, Iorio R, De Carli A et al (2013) An extra-articular procedure improves the clinical outcome in anterior cruciate ligament reconstruction with hamstrings in female athletes. Int Orthop 37(2). doi:10.1007/s00264-012-1571-0

3. Dejour D, Vanconcelos W, Bonin N et al (2013) Comparative study between mono-bundle bone-patellar tendon-bone, doublebundle hamstring and mono-bundle bone-patellar tendon-bone combined with a modified Lemaire extra-articular procedure in 
anterior cruciate ligament reconstruction. Int Orthop 37(2). doi:10.1007/s00264-012-1718-z

4. Chechik O, Amar E, Khashan M et al (2013) An international survey on anterior cruciate ligament reconstruction practices. Int Orthop 37(2). doi:10.1007/s00264-012-1611-9

5. Barenius B, Webster WK, McClelland J (2013) Hamstring tendon anterior cruciate ligament reconstruction: does gracilis tendon harvest matter? Int Orthop 37(2). doi:10.1007/s00264-012-1672-9 -k

6. Li X, Xu CP, Song JQ, Jiang N et al (2013) Single-bundle versus double-bundle anterior cruciate ligament reconstruction: an up-todate meta-analysis. Int Orthop 37(2). doi:10.1007/s00264-0121651-1

7. Suomalainen P, Kannus P, Järvelä T (2013) Double-bundle anterior cruciate ligament reconstruction: a review of literature. Int Orthop 37(2). doi:10.1007/s00264-012-1680-9

8. Zhu W, Lu W, Ou Y et al (2013) Applications of computerized navigation technique for assisting anterior cruciate ligament reconstruction in arthroscopy. Int Orthop 37(2). doi:10.1007/s00264012-1764-6

9. Muller B, Hofbauer M, Wongcharoenwatana J et al (2013) Indications and contraindications for double-bundle ACL reconstruction. Int Orthop 37(2). doi:10.1007/s00264-012-1683-6

10. Arnold MP, Duthon V, Neyret P (2013) Double incision isoanatomical ACL reconstruction: the freedom to place the femoral tunnel within the anatomical attachment site without exception. Int Orthop 37(2). doi:10.1007/s00264-012-1681-8

11. Brown Ch, Spalding T, Robb C et al (2013) Medial portal technique for single-bundle ACL reconstruction. Int Orthop 37(2). doi:10.1007/s00264-012-1772-6

12. Struewer J, Ziring E, Frangen TM et al (2013) Clinical outcome and prevalence of osteoarthritis after isolated anterior cruciate ligament reconstruction using hamstring graft: follow-up after two and ten years. Int Orthop 37(2). doi:10.1007/s00264-0121653-Z

13. Streich N, Reichenbacher S, Barie A et al (2013) Long term outcome of ACL reconstruction with an autologous 4-strand semitendinosus tendon autograft. Int Orthop 37(2). doi:10.1007/ s00264-012-1757-5

14. Feller J, Webster KE (2013) Return to sport following anterior cruciate ligament reconstruction. Int Orthop 37(2). doi:10.1007/ s00264-012-1690-7

15. Vrancken AC, Buma P, van Tienen TG (2013) Synthetic meniscus replacement: a review. Int Orthop 37(2). doi:10.1007/s00264-012$1682-7$

16. Hirschmann MT, Mathis D, Rasch H, Amsler F et al (2013) SPECT/CT tracer uptake is influenced by tunnel orientation and position of the femoral and tibial ACL graft insertion site. Int Orthop 37(2). doi:10.1007/s00264-012-1704-5

17. Hu J, Qu J, Xu D, Zhou J et al (2013) Allograft versus autograft for anterior cruciate ligament reconstruction: an up-to-date metaanalysis of prospective studies. Int Orthop 37(2). doi:10.1007/ s00264-012-1720-5

18. Newman SD, Atkinson HD, Willis-Owen CA (2013) Anterior cruciate ligament reconstruction with the ligament augmentation and reconstruction system: a systematic review. Int Orthop 37(2). doi:10.1007/s00264-012-1654-y

19. Struewer J, Ziring E, Ishaque B et al (2013) Second-look arthroscopic findings and clinical results after polyethylene terephthalate augmented anterior cruciate ligament reconstruction. Int Orthop 37 (2). doi:10.1007/s00264-012-1652-0

20. Boutefnouchet T, Bentayeb M, Qadri Q, Ali S (2013) Long-term outcomes following single-bundle transtibial arthroscopic posterior cruciate ligament reconstruction. Int Orthop 37(2). doi:10.1007/ s00264-012-1609-3 